\title{
Generalized yrast states of a Bose condensate in harmonic trap for a universality class of repulsive interactions
}

\author{
M. S. Hussein and O.K. Vorov \\ Instituto de Fisica, Universidade de Sao Paulo \\ Caixa Postal 66318, 05315-970, \\ Sao Paulo, SP, Brasil
}

(27 February 2001)

\begin{abstract}
For a system of $N$ bosons in a $2 \mathrm{~d}$ harmonic trap with frequency $\omega$, interacting via repulsive forces $V \ll \hbar \omega$, we develop supersymmetric method to find the lowest energy states of rotating Bose condensate as function of two quantum numbers, the total angular momentum and the angular momentum of internal excitations (generalized yrast states). The energies of these condensed vortex states are expressed through the single two-body matrix element of interaction $V$. A broad universality class of the repulsive interactions for which these results hold is described by a simple integral condition on $V$. It includes Gaussian, $\delta$-function and $\log$-Coulomb forces.
\end{abstract}

PACS numbers: 03.75.Fi, 32.80.Pj, 67.40.Db, 03.65.Fd

Typeset using REVTEX 
Creation of traps for cold rarified atoms [1], which has made possible experimental investigation of Bose-Einstein condensation, has stimulated theoretical studies on the systems of weakly interacting bosons confined by parabolic potential [2 6]. Among the important questions about behavior of such systems is their response to rotation and the onset of vorticity [2 [6]. In this context, of great importance is the structure and the spectrum of the ground states of rotating condensate at given angular momentum, the yrast states [2 2 [5].

The particular problem of wide recent interest [3 5] arises in the weak coupling limit, which is hoped [2, to be reached experimentally in nearest future. One considers $N$ spinless bosons in spherical $2 \mathrm{~d}$ harmonic trap [7]. The noninteracting system has equidistant spectrum $\hbar \omega n$ of high degeneracy 田 which grows exponentially with $n$ at $n-N \gg 1$. This degeneracy is related to the number of ways to distribute the total energy $\hbar \omega n$ among the particles. The short-range interactions $V(r)$ between the atoms are assumed weak in the sense that hoppings between different $\hbar \omega n$ levels can be neglected, and the problem still requires nonperturbative solution for the highly degenerate states at single level $\hbar \omega n$, which is similar in spirit to the problem of Landau level for the electrons in magnetic field or to the problem of compound states in an atomic nucleus. As is usual for interacting many-body problems, evaluation of exact ground state is expensive task. So far, the only results for the yrast states in repulsive case were obtained numerically and for simplified $\delta$-forces [5].

In this work, we provide rigorous analytical solution for the ground state as a function of two quantum numbers, the total angular momentum, $L(\leq N)$, and the angular momentum of internal excitations, for a broad class of repulsive interactions. These generalized yrast states include the usual yrast states. The results allow transparent physical interpretation. In fact, the form of the yrast wave functions which was drawn from numerics for the $\delta$ interaction case [5], turns out to be valid in general. The universality class of interactions is described by an explicit sufficiency condition.

For this "single Landau level" problem, the Hamiltonian $(\hbar=m=1)$ is the sum

$$
\tilde{H}=\omega H_{0}+\tilde{V},
$$


where the first term (the energy of degenerate level) comes from the noninteracting Hamiltonian in the parabolic trap, $\sum_{i}^{N}\left(\frac{\vec{p}_{i}^{2}}{2}+\frac{\omega^{2}}{2} \vec{r}_{i}^{2}\right)$, and the second (nontrivial) term $\tilde{V}$ is a properly projected interaction $V=\sum_{i>j} V\left(r_{i j}\right)$, see below. In $2 \mathrm{~d}$, one uses complex $z\left(z^{*}\right)=x \pm i y$ instead of vector $\vec{r}=(x, y)$, and we set $\omega \equiv 1$ for brevity. With $\partial_{ \pm} \equiv \frac{1}{2}\left(\frac{\partial}{\partial x} \mp i \frac{\partial}{\partial y}\right)$, we employ the tetrad of ladder operators $a^{+}, a, b^{+}$and $b$ for each particle,

$$
\begin{gathered}
a^{+}=z / 2-\partial_{+}, \quad b^{+}=z^{*} / 2-\partial_{-}, \\
a=\left(a^{+}\right)^{\dagger}, \quad b=\left(b^{+}\right)^{\dagger}, \quad\left[a, a^{+}\right]=\left[b, b^{+}\right]=1,
\end{gathered}
$$

which raise (lower) the powers of $z_{i}$ and $z_{j}^{*}$ in the preexponentials of many-body wave functions, which are all polynomials times the Gaussian factor $|0\rangle=\exp \left(-1 / 2 \sum\left|z_{k}\right|^{2}\right)$. So, $z_{i}|0\rangle \equiv a_{i}^{+}|0\rangle, z_{j}^{*}|0\rangle \equiv b_{j}^{+}|0\rangle, a_{i}|0\rangle=0, b_{i}|0\rangle=0$, etc. The $2 \mathrm{~d}$ angular momentum is the difference $L=L_{+}-L_{-}$of numbers of "up" and "down" quanta, $L_{+}=\sum_{1}^{N} a_{k}^{+} a_{k}$ and $L_{-}=\sum_{1}^{N} b_{k}^{+} b_{k}$, while the energy of the level in (11) is $H_{0}=L_{+}+L_{-}+N$. The yrast states must have minimum energy at given $L$. Thus, they belong to the subspace with $L_{-}=0$, $L_{+}=L$, spanned by the homogeneous (degree $L$ ) symmetric polynomials , poly ${ }_{S}^{L}\left(a_{i}^{+}\right)|0\rangle$ which do not involve $z^{*}$. The basis is formed by polynomials $\left[l_{1}, l_{2}, \ldots, l_{N}\right]$, obtained by applying the operator of symmetrization over $N$ variables, $P_{S}$, to the monomials $m$ [

$$
\left[l_{1}, l_{2}, \ldots, l_{N}\right] \equiv P_{S} m, \quad m \equiv z_{1}^{l_{1}} z_{2}^{l_{2}} \ldots z_{N}^{l_{N}}
$$

with a given partition of the integer $L=\sum_{n} l_{n}$, 8 . In our case, $L \leq N$, the dimensionality of the basis is $p(L)$, "the number of unrestricted partitions" of integer $L$.

The system enjoys an additional conserved quantity [3, 4]. The collective operators $\mathcal{A}^{+}=$ $\sum_{i=1}^{N} \frac{a_{i}^{+}}{\sqrt{N}}$ and $\mathcal{A} \equiv\left(\mathcal{A}^{+}\right)^{\dagger}$ (with $\left[\mathcal{A}, \mathcal{A}^{+}\right]=1$ ) commute with $\tilde{H}$, and so does the number of collective quanta, $\mathcal{A}^{+} \mathcal{A}$. The mutual eigenfunctions of $\tilde{H}, L$ and $\mathcal{A}^{+} \mathcal{A}=v$ are factorized

$$
\Psi(L, v)=Z^{v} \operatorname{poly} S_{S}^{L-v}\left(\tilde{z}_{i}\right) e^{-\sum \frac{\left|z_{k}\right|^{2}}{2}}
$$

with $\tilde{z}_{i}=z_{i}-Z$ and $Z \equiv \sum_{1}^{N} z_{k} / N$. In (四), the total angular momentum $L$ is redistributed between internal excitations $(J=L-v)$ and the collective motion $(v)$. It is therefore interesting 
to consider the ground state as a function of both $L$ and $v$. We call the eigenvalue of $\mathcal{A}^{+} \mathcal{A}$ seniority for brevity. At given $L$, the allowed values are $v=0,1,2, \ldots, L-2, L$; the value $v=L-1$ is forbidden because $\operatorname{pol}_{S}^{1}\left(z_{i}-Z\right) \equiv 0$. The states with definite seniority, of type (4), can be obtained by applying the projector $\mathcal{P}_{v}$ [10] to (3).

In a given sector $L$, the projected [10] interaction $\tilde{V}$ in (10) is given by [11,12]

$$
\tilde{V}=P_{L_{-}=0} P_{L_{+}=L} V P_{L_{+}=L} P_{L_{-}=0} .
$$

In order to evaluate (5), we use the Fourier representation

$$
V=\sum_{i>j} \int_{-\infty}^{\infty} d^{2} \vec{q} e^{i\left[q_{-}\left(a_{i j}^{+}+b_{i j}\right)+q_{+}\left(b_{i j}^{+}+a_{i j}\right)\right]} V_{q}
$$

where $V_{q}=\int_{0}^{\infty} r d r J_{0}(r|q|) V(r) /(2 \pi)$ is the $2 \mathrm{~d}$ Fourier transform of the potential $V(r)$, with $J_{0}$ the Bessel function [9] and $q_{ \pm}=\left(q_{x} \pm i q_{y}\right) / \sqrt{2}$. The two-particle combinations $a_{i j}^{+} \equiv \frac{1}{\sqrt{2}}\left(a_{i}^{+}-a_{j}^{+}\right), b_{i j}^{+} \equiv \frac{1}{\sqrt{2}}\left(b_{i}^{+}-b_{j}^{+}\right)$etc. came from resolving $x_{i(j)}$ and $y_{i(j)}$ from (2). Substituting (66) in (5) and using Baker-Hausdorf relation $e^{a^{+}-a}=e^{\frac{\left[a^{+}, a\right]}{2}} e^{a^{+}} e^{-a}$, we obtain expansion of $\tilde{V}$ in terms of the two-particle operators $B^{k} \equiv \sum_{j>i}^{N} a_{i j}^{\dagger k} a_{i j}^{k}$,

$$
\begin{array}{r}
\tilde{V}=\sum_{k=0}^{2[L / 2]}(-1)^{k} s_{k} B^{k}=\sum_{k=0}^{[L / 2]} V_{k}, \\
V_{k} \equiv s_{2 k}\left(B^{2 k}-B^{2 k-1}\right)+\left(s_{2 k+2}-s_{2 k+1}\right) B^{2 k+1} .
\end{array}
$$

The highest possible order of $B^{k}$ is $L$ for $L$ even, and $L-1$ for $L$ odd; $B^{0} \equiv N(N-1) / 2$. The shape of potential $V(r)$ is described by the integrals with Kummer function $M$ [9], 13]

$$
s_{k} \equiv \int_{0}^{\infty} \frac{d t}{k !} M(k+1,1,-t) V(\sqrt{2 t}) .
$$

Expansion (7]) is exact for any interaction $V(r)$ whose moments $s_{k}$ are finite [11,12].

Regular methods to obtain exact ground state without solving the whole spectrum are not available. We use the approach [12] which we loosely nicknamed "supersymmetry" [14]. Suppose that the Hamiltonian can be written as a sum,

$$
\tilde{V}=V_{0}+V_{S}
$$


such that

(a) the first term, $V_{0}$, is simple, and one can find out its ground state $\left.\mid 0\right)$ with eigenvalue $\mathcal{E}_{\text {min }}$ (possibly degenerate). If the second term, $V_{S}$, has the two "supersymmetric" properties:

(b) $V_{S}$ annihilates the state $\left.\mid 0\right)$, so $\left.V_{S} \mid 0\right)=0$,

(c) $V_{S}$ is non-negative definite, $V_{S} \geq 0$, (it does not have negative eigenvalues),

then the state $\mid 0$ ) will still be the ground state for the full Hamiltonian $V_{0}+V_{S}$, with the same eigenvalue $\mathcal{E}_{\text {min }}$. Indeed, (b) implies that $\left.\mid 0\right)$ is an eigenstate for the sum $V_{0}+V_{S}$ with its eigenvalue intact. As one knows form linear algebra, if an Hermitean operator $V_{0}$ is perturbed by a non-negative definite Hermitean operator $V_{S}$, the eigenvalues can only increase (see, e.g. [15]). This means that the states other than |0) can gain energy [Cf. (b)]. As $\mid 0)$ is already the ground state for $V_{0}$, it will be the same for $V_{0}+V_{S}$ [16, 17.

The same arguments apply to the case with additional conserved quantum number $v$, such as $\left[v, V_{0}\right]=\left[v, V_{S}\right]=0$. In the above scheme, the single state $\left.\mid 0\right)$ is replaced by the set of states $\mid L, v)$, each having the property (a) and (b) in their $v$-sectors. This is illustracted in Fig.1, where the spectrum of $V_{0}$ is taken degenerate in each $v$-sector.

In our case, the supersymmetric triad $V_{0}, V_{S}$ and $\left.\mid 0\right)$ can be established by inspecting action of terms $V_{k}$ in (7) on the states of partition basis (3). While the first term $V_{0}$, by virtue of identity $N \sum_{i}^{N} a_{i}^{+} a_{i}=\frac{1}{2} \sum_{i, j}^{N}\left(a_{i}^{+}-a_{j}^{+}\right)\left(a_{i}-a_{j}\right)+\left(\sum_{i}^{N} a_{i}^{+}\right)\left(\sum_{i}^{N} a_{j}\right)$, is reduced to a combinations of quantum numbers,

$$
V_{0}=(N / 2)\left[(N-1) s_{0}-\left(L-\mathcal{A}^{+} \mathcal{A}\right)\left(s_{1}-s_{2}\right)\right]
$$

the simplest state $|L\rangle$ in (3) with partition $[1,1, \ldots 1]$ is annihilated by the remainder of the Hamiltonian,

$$
|L\rangle=P_{S} a_{1}^{+} a_{2}^{+} \ldots a_{L}^{+}|0\rangle, \quad\left(\tilde{V}-V_{0}\right)|L\rangle=0,
$$

Indeed, $\left[a_{12}^{2}, a_{1}^{+} a_{2}^{+}\right]=-2\left(a_{12}^{+} a_{12}+1\right)$ and thence $a_{12}^{k(>2)} a_{1}^{+} a_{2}^{+} a_{3}^{+} \ldots a_{L}^{+}|0\rangle=0$ and $B^{k>2}|L\rangle=0$. From the same commutator, we have $\left[a_{12}^{+2} a_{12}^{2}, a_{1}^{+} a_{2}^{+}\right]|0\rangle=\left[a_{12}^{+} a_{12}, a_{1}^{+} a_{2}^{+}\right]|0\rangle=-a_{12}^{+2}|0\rangle$, and thus $\left(B^{2}-B^{1}\right)|L\rangle=0$. Substitution $z_{i}=\tilde{z}_{i}+Z$ [see (4) $]$ transforms $|L\rangle$ to a sum 


$$
|L\rangle=\left[P_{S} \tilde{z}_{1} \tilde{z}_{2} \ldots \tilde{z}_{L}+\ldots+Z^{L-2} P_{S} \tilde{z}_{1} \tilde{z}_{2}+Z^{L}\right]|0\rangle=\sum_{v} \mathcal{P}_{v}|L\rangle
$$

of exactly $L$ seniority observing states of the form (4), each being the eigenvector of $\mathcal{A}^{+} \mathcal{A}$ and therefore of $V_{0}$, with the eigenvalue (10). We notice that each $v$-sector is represented by a single term in (12), identified with $\mathcal{P}_{v}|L\rangle$.

The spectrum of $V_{0}$ (see Fig.1.) consists of $L$ equidistant (except $\left.v \neq L-1\right), g(v)$-fold degenerate levels with energies (10); $g(v)=p(L-v)-p(L-v-1)$ for $v \leq L-2$ and $g(L)=1$. Each $v$-level contains one and only one state $\mid L, v)=\mathcal{P}_{v}|L\rangle$ from the sum (12) Therefore, the set of states $\mid L, v)$ obey the criterion (a) with the operator $V_{0}$. The property (b) with $V_{S} \equiv \tilde{V}-V_{0}$ holds by virtue of (11). In particular, (a) together with (b) mean that $\left.\mid L, v\right)$ are the eigenvectors of $\tilde{V}=V_{0}+V_{S}$ with eigenvalues (10). This holds for any interaction $V(r)$.

The supersymmetric representation for the triad $V_{0}, V_{S}$ and $\left.\mid L, v\right)=\mathcal{P}_{v}|L\rangle$ would be complete if we succeeded to prove non-negative definiteness $V_{S} \geq 0$ of the remainder of the Hamiltonian $\tilde{V}-V_{0}$, criterion (c). So far, we did not specify form of the interaction $V(r)$ in ([7). We will study now general case and specify the class of potentials which have $V_{S} \geq 0$.

We have to check signs of all the eigenvalues of $V_{S}$ in the partition space (3)). To avoid solving the whole spectrum, in the space of symmetrized states, we use the following trick. By definition, the nonzero eigenvalues of $V_{S}$ in the space (3) coinside with the nonzero eigenvalues of $P_{S} V_{S} P_{S}$ in the full space of monomials $m$ in (3). This latter space has dimensionality much higher than $p(L)$ and it includes wave functions of all possible symmetries, including boson sector (fully symmetric), fermion sector (fully antisymmetric) etc. In this extended space, the analysis of signs of eigenvalues is however crucially simplified, while the contributions from the symmetric sector can be accurately separated. Using the symmetry of $V_{S}$ under permutations of particles, we write

$$
P_{S} V_{S} P_{S}=P_{S} \sum_{i>j} V_{S, i j} P_{S}=(N / 2)(N-1) P_{S} V_{S, 12} P_{S}
$$

where $V_{S, i j}$ is the contribution from pair of particles $i, j$ to $V_{S}$ [Cf.(17,8, 90)]. In order to see that $P_{S} V_{S} P_{S} \geq 0$, it is sufficient to show that $V_{S, 12} \geq 0$, because application of any projector $P_{S}$ 
from both sides in (13) can add new zero eigenvalues, but can not add negative eigenvalues. This follows from the known "inertia theorem" of linear algebra [15]. Now, we study the eigenvalues of $V_{S, 12}$. Let $\pi_{12}$ be the operator of permutation of variables 1 and 2 . The triad

$$
T=\left\{\pi_{12}, \quad a_{12}^{+} a_{12}, \quad V_{S, 12}\right\}
$$

forms a set of mutually commuting operators. Indeed, $V_{S, 12}$ is expressed in terms of $B_{12}^{k}=$ $a_{12}^{+k} a_{12}^{k}[$ see (7) $]$. As $\left[a_{12}, a_{12}^{+}\right]=1$, any $B_{12}^{k}$ can be expressed in terms of $a^{+}{ }_{12} a_{12}$, using boson calculus formula $a_{12}^{+k} a_{12}^{k}=a_{12}^{+} a_{12}\left(a_{12}^{+} a_{12}-1\right) \ldots\left(a_{12}^{+} a_{12}-k+1\right)$. The triad $T$ is diagonalized simultaneously right in the basis of monomials $m \equiv\left\{z_{1}^{l_{1}} z_{2}^{l_{2}} z_{3}^{l_{3}} \ldots z_{N}^{l_{N}}\right\}$, (3) with the only substitutions $z_{1} \rightarrow \frac{1}{\sqrt{2}}\left(z_{1}-z_{2}\right), z_{2} \rightarrow \frac{1}{\sqrt{2}}\left(z_{1}+z_{2}\right)$. In this basis, the eigenvalues of the triad $T$ depend only on $l_{1}$ in the subfactor $\left[\frac{1}{\sqrt{2}}\left(z_{1}-z_{2}\right)\right]^{l_{1}}$ of monomial $m$, they are $\left\{(-1)^{l_{1}}, l_{1}, \quad \lambda_{l_{1}}\right\}$, respectively. Using Eqs.(77) and (8) and the summation formula $\sum_{k=0}^{N} \frac{(-1)^{k} N !}{k !(N-k) !} M(k+1,1,-t)=\frac{e^{-t} t^{N}}{N !}$, we obtain $\lambda_{l_{1}}=\int_{0}^{\infty} r d r V(r) f_{l_{1}}(r)$ with $f_{l_{1}}(r)=e^{-r^{2} / 2}\left[\frac{r^{2 l_{1}}}{2^{l_{1} l_{1} !}}-1+\frac{l_{1}}{4}\left(2-\frac{r^{4}}{4}\right)\right]$. The eigenvalue $(-1)^{l_{1}}$ of $\pi_{12}$ helps now to separate out states with wrong symmetry: the eigenvectors with $l_{1}$ odd are antisymmetric in $z_{1}, z_{2}$, and the projector $P_{S}$ in (13) kills them all. All even values of $l_{1}(\leq L)$ can contribute to the bosonic sector, and the corresponding $\lambda$ 's must be checked.

Now, inequality $\lambda_{2 n} \geq 0$ for any $2 n \leq L$ is the sufficient condition which defines the class of potentials for which $V_{S}$ is non-negative definite, and the triad $V_{0}, V_{S} \equiv \tilde{V}-V_{0}$ and $\left\{\mathcal{P}_{v}|L\rangle\right\}$ obeys the supersymmetric representation with properties (a), (b) and (c), with $\mathcal{P}_{v}|L\rangle$ being the ground state in its sector $L, v$ with the energy $\mathcal{E}_{\min }$ equal to eigenvalue of $V_{0}$.

With this criterion and (12) and (10) at hand, we can formulate very general result: For any bona fide two-body potential $V(r)$ which satisfies the integral condition

$$
\begin{array}{r}
\int_{0}^{\infty} V(\sqrt{2 t}) e^{-t}\left[\frac{t^{2 n}}{(2 n) !}-1+n\left(1-\frac{t^{2}}{2}\right)\right] \geq 0 \\
\text { for any } n \leq L^{\prime} / 2,
\end{array}
$$

the eigenstates of the Hamiltonian $\tilde{H}$ with minimal energies at given pair of $v$ and $L\left(\leq \min \left\{L^{\prime}, N\right\}\right)$ have universal form

$$
\mid L, v)=e^{-\frac{1}{2} \sum\left|z_{i}\right|^{2}} Z^{v}\left(\frac{\partial}{\partial Z}\right)^{N-L+v} \prod_{k=1}^{N}\left(z_{k}-Z\right),
$$




$$
Z \rightarrow \frac{1}{N} \sum_{i=1}^{N} z_{i}
$$

with the energies given by the simple moments of $V(r)$,

$$
\begin{gathered}
\mathcal{E}_{\text {min }}(L, v)=L+N+\frac{N(N-1)}{2} \mathcal{V}_{0}+\frac{\mathcal{V}_{0}-\mathcal{V}_{1}}{2} N(v-L) \\
\mathcal{V}_{0}=\int_{0}^{\infty} d t e^{-t} V(\sqrt{2 t}), \quad \mathcal{V}_{1}=\int_{0}^{\infty} d t e^{-t}\left(\frac{1}{2}+\frac{t^{2}}{4}\right) V(\sqrt{2 t})
\end{gathered}
$$

which are equal to expectation values of interaction between two bosons both in the ground $\mathcal{V}_{0}=\langle 00|V| 00\rangle$ and the first excited state of oscillator $\mathcal{V}_{1}=\langle 11|V| 11\rangle$, respectively 18 .

At fixed $L$, we have exactly $L$ such equidistant generalized yrast states, marked by $v=$ $0,1,2, \ldots, L(v \neq L-1)$, see Fig.1. Each such state is the "ground state" in the sector $L, v$ (of course, there are other states in each sector with higher energies).

The usual yrast states minimize $\mathcal{E}_{\min }(L, v)$ with respect to seniority, $v$. From (16), they can have either $v=0$ or $v=L$. As can be seen, in the domain of validity (14) the first option is usually realized. Inequality $\left(\mathcal{V}_{0}-\mathcal{V}_{1}\right)(v-L) \leq 0$ means that internal rotational excitations are energetically favorable, once the interaction energy between two bosons in the state $z|0\rangle$ is less, then in the state $|0\rangle$. Physically, the yrast wave functions (15) with $v=0$ correspond to condensation to a vortex, rotating around the "center-of-mass", as discussed in [3]. The maximum seniority states $v=L$, which correspond to purely collective rotation [4] with no internal excitations, were shown [3] to be energetically favorable for attractive $\delta$-forces.

Consider repulsive $\left(U_{0} \geq 0\right)$ Gaussian interaction, $V(r)=U_{0} \frac{e^{-r^{2} / R^{2}}}{\pi R^{2}}$, whose range $R$ can be varied from zero to infinity. From (14), we have $\lambda_{2 n}=\frac{U_{0}}{\pi\left(2+R^{2}\right)}\left[\left(\frac{R^{2}}{2+R^{2}}\right)^{2 n}-1+4 n \frac{1+R^{2}}{\left(2+R^{2}\right)^{2}}\right] \geq 0$, so (14) holds for any $R$, and the spectrum is $\mathcal{E}_{\min }=L+N+\frac{U_{0}}{\pi\left(2+R^{2}\right)}\left[N(N-1) / 2-\frac{\left(1+R^{2}\right)}{\left(2+R^{2}\right)^{2}} N(L-\right.$ $v)$. The yrast states have $v=0$. In particular, in the zero range limit, $R \rightarrow 0$, we have the $\delta$-function repulsive interaction $V=U_{0} \delta(\vec{r})$. We have $\lambda_{l_{1}}=\frac{U_{0}}{2 \pi}\left[\delta_{l_{1}, 0}+\frac{l_{1}}{2}-1\right]$. It is seen that while $\lambda_{1}=-1 / 2$, for any $l_{1}=2 n$ even the condition $\lambda_{2 n} \geq 0$ (14) holds. The energies (16) are $\mathcal{E}_{\text {min }}=L+N+\frac{U_{0}}{8 \pi} N(2 N-L+v-2)$. Yrast states have $v=0$, and $\mathcal{E}_{\text {min }}(L, 0)$ agrees with that obtained numerically [5], see also [19].

For the 2 d Coulomb interaction $V=U_{0} \log (1 / r)$ with $U_{0} \geq 0$, we have $\lambda_{2 n}=\frac{U_{0}}{4}[3 n-$ 
$1 / n+2 \psi(1)-2 \psi(2 n)] \geq 0$, where $\psi$ is digamma function, so (14) holds, and $\mathcal{E}_{\min }=$ $L+N-\frac{U_{0} N}{4}[(\log (2)-\gamma)(N-1)-(3 / 4)(v-L)]$ with $\gamma=-\psi(1)=0.57721 \ldots$ the Euler constant. The yrast states correspond to $v=0$.

Let us see, that condition (14) imposes only weak restrictions on the repulsive forces $V(r)$. Indeed, at small $r \simeq 0$, the factor-function $f_{2 n}(r)$ in $\int_{0}^{\infty} r d r f_{2 n}(r) U(r)$ in (14) approach positive constant values, $n-1$ and $d f_{n} /\left.d r\right|_{r=0}=0$, while the first node of the functions $f_{2 n}(r)$ occurs at $r_{0}=\sqrt{2 \sqrt{12-2 \sqrt{30}}} \simeq 1.43$ which is of order of the oscillator length, in our units ( $\hbar=m=\omega=1$ ). Therefore, for any short range (as compared to the characteristic length of the trap) interaction, the condition (14) reduces to

$$
\int d^{2} \vec{r} V(r) \geq 0
$$

Thus, the results (15,16) hold for short-range interactions, which are repulsive on average.

The condition (14) holds even for many long-range interactions. This is seen from behavior of function $f_{4}(r)(n=0$ and $n=1$ give $f \equiv 0)$ which is positive at $r<r_{0}$ and $r>r_{1}=\sqrt{2 \sqrt{12+2 \sqrt{30}}} \simeq 3.10$, and $f_{4}$ is negative only in the interval $r_{0} \leq r \leq r_{1}\left(f_{n}(r)\right.$ for higher $n$ behave similarly). It is clear that (14) holds, if $V(r)$ decreases monotonically and fast enough, as is the case for the long range Gaussian and Coulomb forces. One can therefore summarize that condition holds for any physically meaningful repulsive interaction.

To conclude, in the problem of weakly interacting Bose condensate in $2 \mathrm{~d}$ trap, we developed an exact expansion for the interaction to powers of ladder operators. A supersymmetric representation for this interaction has been developed to obtain the states with minimum energy at given angular momentum and seniority (generalized yrast states, including usual yrast states). The energies of these condensed vortex states are given by simple integral moments of the interaction potential. A broad universality class of the repulsive interactions for which these results are valid is described explicitly.

Further applications of these results are straightforward. In view of [7], the threedimensional case can be done using the same method. It is also interesting to study region of higher angular momenta $L>N$, where the structure of the basis polynomials will be 
changed [8], while the numerical studies indicate signs of phase transition [5]. The method of "supersymmetric decomposition" developed here is not restricted to this particular problem and can be applied to fermions and even to the particles with parastatistics. The work was supported by FAPESP. 


\section{REFERENCES}

[1] M.H. Anderson, J.N. Ensher, M.R. Matthews, C.E. Weiman, and E.A. Cornell, Science,, 269, 198 (1997).

[2] D.A.Butts and D.S. Rokhsar, Nature 397, 327 (1999).

[3] N. K. Wilkin, J. M. Gunn, and R. A. Smith, Phys. Rev. Lett. 80, 2265 (1998).

[4] B. Mottelson, Phys. Rev. Lett. 83, 2695 (1999).

[5] G. F. Bertsch and T. Papenbrock, Phys. Rev. Lett. 83, 5412 (1999).

[6] F. Dalfovo, S. Giorgini, L. P. Pitaevskii, and S. Stringari, Rev.Mod.Phys. 71, 463 (1999).

[7] As was shown in [4,5], the $3 \mathrm{~d}$ case reduces to $2 \mathrm{~d}$ case in the weak coupling limit.

[8] B. Higman, Applied group-theoretic and matrix methods, (New York, Dover, 1964).

[9] M. Abramowitz and I.A. Stegun, Handbook of Mathematical Functions, (National Bureau of Standards, 1964).

[10] We use the standard number-of-quanta projectors $\mathcal{P}_{v}=\frac{1}{2 \pi} \int_{0}^{2 \pi} d \phi e^{i \phi\left(v-\mathcal{A}^{+} \mathcal{A}\right)}, P_{L}=$ $\frac{1}{2 \pi} \int_{0}^{2 \pi} d \alpha e^{i \alpha\left(L-\sum_{i} a_{i}^{+} a_{i}\right)}$ etc.

[11] M.S. Hussein and O.K. Vorov, New results for the yrast spectra of weakly interacting identical bosons, June 2000, Abstr. subm. to the Int. Symposium Nuclei and Nucleons 2000, Darmstadt; Analytical results for the yrast spectra of interacting bosons, July 2000, Abstr. submitted to the Annual Meet. of the Brazilean Phys. Society.

[12] M.S. Hussein and O.K. Vorov, subm. to Phys. Lett. A.

[13] For $k$ integer, it can be related to Laguerre polynomials $M(k+1,1,-t)=e^{-t} L_{n}^{0}(t)$.

[14] There is a tiny formal point in common with "factorization method", E.Schrödinger, Proc.R.Irish.Acad. A46,9 (1940), and the supersymmetry, E.Witten, Nucl. Phys. B188, 513 (1981); F.Cooper, J.N.Ginocchio, and A.Khare, Phys. Rev. D36,2458 (1986). 
[15] G.W.Stewart and J.-G. Sun, Matrix Perturbation Theory, (Academic Press, New York, 1990).

[16] A trivial example is the $1 \mathrm{~d}$ harmonic oscillator with $V=1 / 2+a^{+} a$. The state $\left.\mid 0\right)$, such as $a \mid 0)=0$, is "the ground state" for $V_{0} \equiv 1 / 2$ because any other state $\left.\mid n\right)$ has the same eigenvalue $1 / 2$. The operator $V_{S}=a^{+} a, V_{S} \geq 0$ acts as perturbation, removing this degeneracy and leaving $\mid 0$ ) with lowest eigenvalue of $V$ [17].

[17] In principle, this does not exclude possibility that the ground state can still be degenerate, if it was such for $V_{0}$. It is possible to show [12] that in our case the ground states of $\tilde{V}$ are not degenerate.

[18] This does not mean that (16) can be obtained as an expectation value over a simple state with only two oscillator levels occupied; in fact, the eigenstates (15) are much more complicated in the second quantization representation.

[19] When the paper was in preparation, we have learned on recent works [20] where the states (15) with $v=0$ were shown to belong to the eigenspace for the case of $\delta$-interaction. Generalization on the case $v \neq 0$ was considered in the last work of Ref. [20].

[20] R.A. Smith and N.K. Wilkin, Phys. Rev. A62, 061602 (2000); A.D. Jackson and G.M. Kavoulakis, Phys. Rev. Lett. 85, 2854 (2000); T. Papenbrock and G.F. Bertsch, J. Phys. A34, 603 (2001) [ cond-mat/0008286]. 


\section{Figure Captions}

Fig.1. Illustraction of supersymmetric decomposition. The spectrum of $V_{0}$ (left) is sequence of degenerate levels, labeled by the conserved quantum number $v$. The spectrum of $V_{0}+V_{S}$ is shown on the right. Supersymmetric perturbation $V_{S} \geq 0$ splits each level, pushing the states up and leaving the lowest energy in each $v$-sector intact. 
FIGURE1 Hussein \& Vorov "Generalized yrast..."
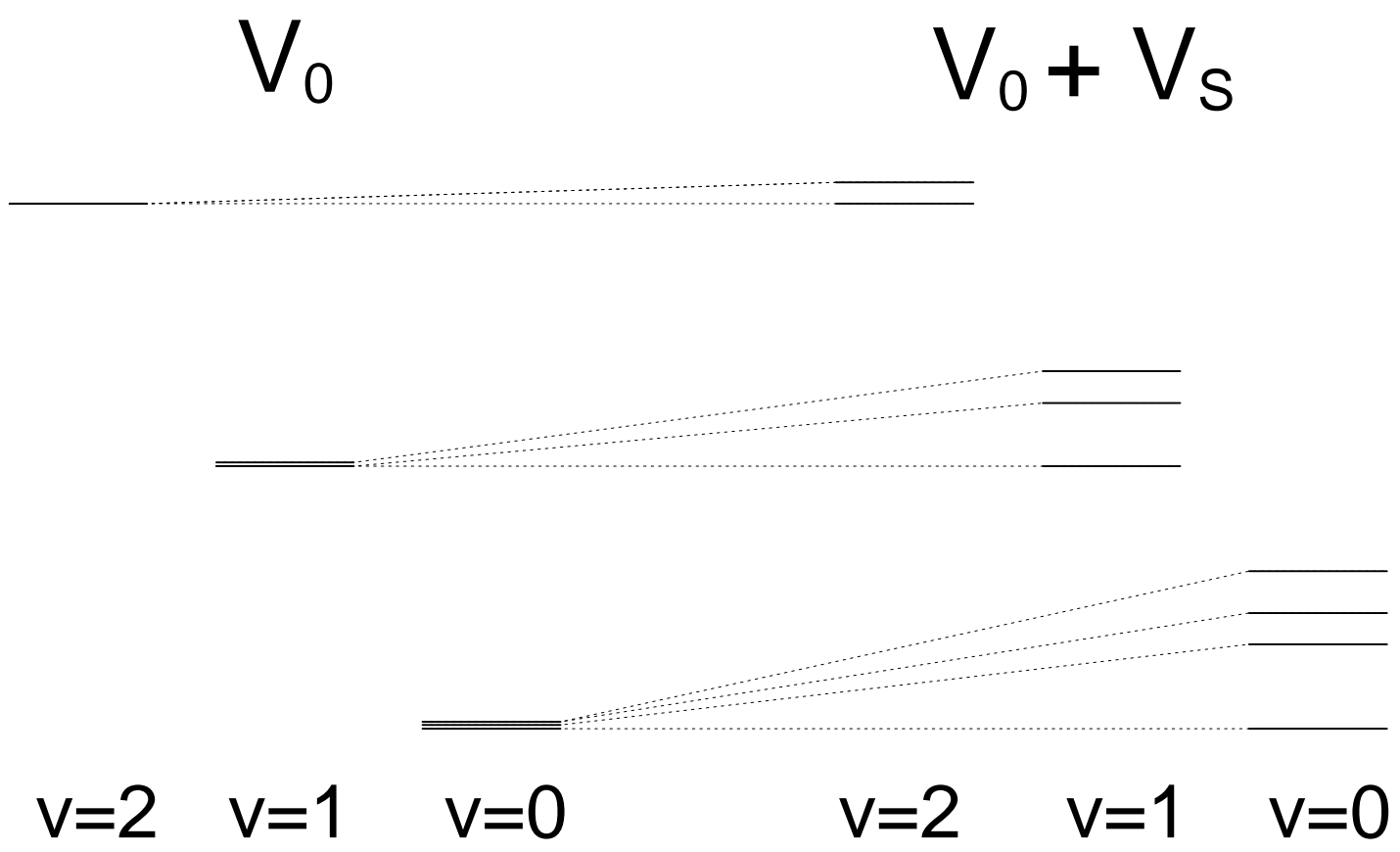